\title{
Isolation of novel murine maternal mRNAs regulated by cytoplasmic polyadenylation
}

\author{
Fernando J. Sallés, ${ }^{1}$ Andrew L. Darrow, ${ }^{1,2}$ Marcia L. O'Connell, ${ }^{1,3}$ and Sidney Strickland ${ }^{1}$ \\ ${ }^{1}$ Department of Pharmacology, State University of New York at Stony Brook, Stony Brook, New York 11794-8651 USA
}

\begin{abstract}
The cytoplasmic polyadenylation element (CPE) is an AU-rich sequence in the $3^{\prime}$-untranslated region of many stored maternal mRNAs. The CPE directs the meiotic maturation-specific cytoplasmic polyadenylation and translational activation of these dormant mRNAs in Xenopus. The work presented here demonstrates that the CPE controls a similar regulation in mouse oocytes and utilizes the information to isolate novel maternal mRNAs by polymerase chain reaction (PCR). A degenerate CPE primer was used in an anchored PCR reaction with cDNAs from primary mouse oocytes. Clones were identified that contained the canonical polyadenylation signal AATAAA. A novel PCR test was then used to determine the polyadenylation state of the respective mRNAs before and after meiotic maturation. Two mRNAs, OM-1 and OM-2, are cytoplasmically polyadenylated upon maturation. Another mRNA is not polyadenylated during maturation, although it contains multiple CPE-like elements, indicating that this sequence element is not sufficient for adenylation during this time. Microinjection into primary oocytes of antisense oligodeoxynucleotides directed against OM-1 destroys the mRNA but does not appear to interfere with maturation in vitro. These experiments identify two novel maternal mRNAs and establish a simple strategy for isolating other maternal messages that control meiotic maturation, fertilization, and early mouse development.
\end{abstract}

[Key Words: Oocyte maturation; meiotic maturation; translational control; anchored PCR; maternal-effect genes]

Received March 2, 1992; revised version accepted April 14, 1992.

Maternal control of early embryogenesis was first recognized as a principle of metazoan development $\sim 100$ years ago (Davidson 1976). Molecular analysis of this principle has been useful in exploring genetic control of early development. For example, in Drosophila, isolation of mutant flies whose homozygous female offspring are sterile has allowed the identification of numerous maternal-effect genes (Nüsslein-Volhard et al. 1987). Detailed analysis of these genes and their functions has revolutionized our molecular understanding of complex morphogenetic processes in insects. Such analyses, however, are not practical in mammals because of the low number of mutants that can be isolated and the lengthy generation time.

In all animals studied, maternal control of development is exerted, at least in part, via translational activation of dormant mRNAs that accumulate in the cytoplasm of the oocyte. These messages are translated when required at meiotic maturation, fertilization, or early embryogenesis (Davidson 1976). The translational

Present addresses: ${ }^{2}$ Department of Microbiology and Immunology, Duke Medical Center, Durham, North Carolina 27710 USA; ${ }^{3}$ Laboratory of Molecular Genetics, National Institutes of Health, Bethesda, Maryland 20892 USA. activation of these stored maternal mRNAs is controlled predominantly by cytoplasmic polyadenylation (Rosenthal et al. 1983; for review, see Rosenthal and Wilt 1987; Dworkin and Dworkin-Rastl 1990; Wickens 1990; Richter 1991). This polyadenylation is variable in terms of its timing, extent, and effect on the stability of specific mRNAs. Despite the differences in regulatory details, however, translational activation of dormant mRNAs by cytoplasmic polyadenylation is a unifying theme of maternal control.

Translational activation of dormant maternal mRNAs appears to be regulated by a $3^{\prime}$-untranslated sequence designated the cytoplasmic polyadenylation element (CPE). A CPE consensus sequence has been defined in Xenopus as UUUUUAU (Fox et al. 1989; McGrew et al. 1989) and is located upstream of the canonical nuclear polyadenylation signal, AAUAAA. Cytoplasmic polyadenylation upon meiotic maturation requires both the CPE sequence and AAUAAA (Fox et al. 1989; McGrew et al. 1989; Vassalli et al. 1989; McGrew and Richter 1990). In Xenopus, the CPE is recognized by factors that presumably regulate the polyadenylation of the message (Paris and Richter 1990; Paris et al. 1991). These CPEbinding proteins, in conjunction with other factors and/ or sequence determinants in the 3 -untranslated region 
(3' UTR), are likely to account for the differences in the regulation of specific mRNAs.

The regulation of cytoplasmic polyadenylation is less well characterized in mammals. Several dormant murine maternal mRNAs have been identified (Huarte et al. 1987; Mutter et al. 1988; Paynton et al. 1988). One of these, the mRNA for tissue plasminogen activator $(t-$ PA), is stored in the cytoplasm of primary mouse oocytes with a short (40-nucleotide) poly(A) tail. Upon meiotic maturation, the poly $(\mathrm{A})$ tail is elongated by $400-500$ nucleotides, and the message begins to be translated (Huarte et al. 1985, 1987). It is known that sequences in the 3' UTR of the mRNA, along with AAUAAA, are necessary for this elongation and that polyadenylation is sufficient to allow translation (Strickland et al. 1988; Vassalli et al. 1989). The present work identifies a CPE in this mRNA that regulates its cytoplasmic polyadenylation.

To further our understanding of maternal developmental control in mammals, we have exploited regulatory aspects of cytoplasmic polyadenylation to identify novel murine maternal mRNAs. A degenerate primer homologous to the CPE consensus sequence was used to amplify oocyte cDNAs that contain this sequence near their $3^{\prime}$ ends. In this manner, cDNA clones containing a CPE were generated. These clones were analyzed by an anchored PCR technique that can assess differences in the polyadenylation state of an mRNA in a single oocyte. Injection of RNA fragments into oocytes was also used to determine whether the CPE-containing sequences direct polyadenylation. This strategy has identified at least two novel mRNAs that are regulated in a manner similar to other dormant mRNAs.

\section{Results}

Identification of the sequence requirements for murine maternal mRNA cytoplasmic polyadenylation

To identify more precisely the sequence requirements for the cytoplasmic polyadenylation of t-PA mRNA, 3' deletions were made in a cDNA clone containing 359 nucleotides of the $3^{\prime}$ UTR (Fig. 1A). These deletion mutants were then used to prepare transcripts with shortened portions of the 3' UTR located upstream of the nuclear polyadenylation signal, AAUAAA, which is also necessary for cytoplasmic polyadenylation in the mouse (Vassalli et al. 1989). Primary mouse oocytes were cytoplasmically injected with these radiolabeled transcripts and allowed to undergo meiotic maturation. The RNA was isolated and analyzed by PAGE and autoradiography. An RNA (from mutant $\Delta 10$ ) with 326 nucleotides of $3^{\prime}$ UTR sequence was not polyadenylated in secondary oocytes, whereas a transcript with 338 nucleotides (mutant $\Delta 24$ ) was (Fig. 1B). These deletion mutants define a 12-nucleotide sequence that is indispensable for t-PA mRNA polyadenylation. A region of this sequence (UUUUAAU) bears strong similarity to CPE sequences shown to be functional in Xenopus oocytes (Fox et al. 1989; McGrew et al. 1989).

A 20-nucleotide oligomer containing the presumptive $\mathrm{CPE}$ (Fig. 1A) was used to generate clones containing one
A
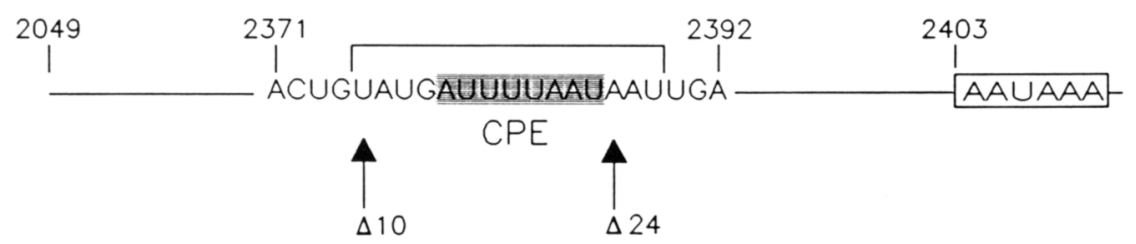

B

$\Delta 10$

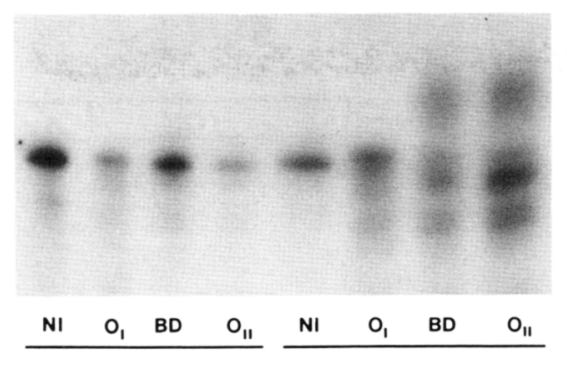

C

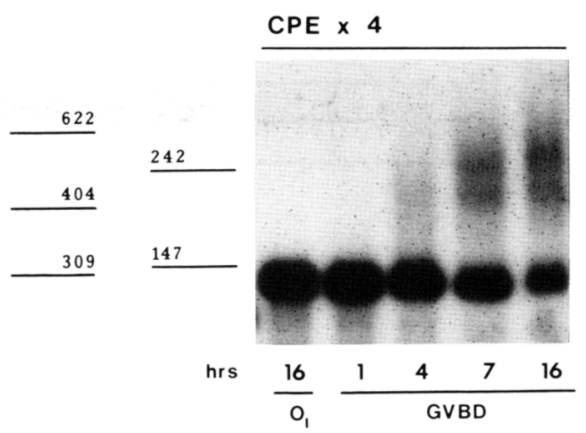

Figure 1. Identification of a $\mathrm{CPE}$ in mouse t-PA mRNA. (A) Schematic representation of the 3' UTR of t-PA mRNA from nucleotide 2049 to 2403 (Rickles et al. 1988). When fused to AAUAAA, an RNA of this sequence is efficiently polyadenylated upon injection into the cytoplasm of maturing oocytes $\left(\mathrm{O}^{\prime}\right.$ Connell 1989). The shaded sequence represents a core CPE based on comparison with Xenopus elements (McGrew et al. 1989; Wickens 1990). (B) Denaturing PAGE analysis of radiolabeled deletion transcripts. Deletion clones $\Delta 10$ and $\Delta 24$ had nucleotide 2049 as their $5^{\prime}$ termini and a $3^{\prime}$ t-PA terminus, as indicated by the arrows in $A$, fused to AAUAAA. (Left lanes) $\Delta 10$ RNA was injected into oocytes; (right lanes) $\Delta 24$ RNA was injected. (NI) Noninjected RNA; $\left(\mathrm{O}_{\mathrm{I}}\right)$ oocytes cultured overnight in the presence of CAMP to prevent maturation; (BD) oocytes cultured overnight that had undergone germinal vesicle breakdown; $\left|\mathrm{O}_{\mathrm{II}}\right|$ oocytes that matured to the secondary oocyte stage. For $\Delta 10$ RNA, 8 oocytes/ lane; for $\Delta 24$ RNA, 12 oocytes/lane. $(C)$ The sequence bracketed in $A$ was tested for its ability to confer polyadenylation when four copies were inserted into a random RNA sequence (CPE $\times 4$ ). Denaturing PAGE analysis of radiolabeled CPE $\times 4$ RNA. (hr) Hours postinjection, (GVBD) oocytes that had matured for the indicated time to the germinal vesicle breakdown stage. All samples have 10 oocytes per lane. 
to four copies of this sequence upstream of AAUAAA. Radiolabeled RNA transcripts were prepared and injected into primary oocytes. RNAs from oocytes that were allowed to mature from 1 to $16 \mathrm{hr}$ were then isolated and analyzed by denaturing PAGE. Transcripts that lacked this sequence were not polyadenylated (data not shown). When the CPE was present, the RNAs were polyadenylated during meiotic maturation (four copies, Fig. $1 C_{\text {; }}$ one to three copies, data not shown). The efficiency of transcript polyadenylation was lower than that observed with the wild-type sequence, which is completely converted to the elongated form in a similar assay (Vassalli et al. 1989). This decrease may be caused by the absence of sequence elements or a structural conformation important for maximal efficiency in the injected CPE-containing fragment. These experiments identify a sequence that is functional as a CPE in mouse oocytes.

\section{Use of the CPE to clone mouse maternal messages}

The approach utilized to identify stored maternal mRNAs (Fig. 2) relies on the conserved nature of the CPE. A PCR primer homologous to the t-PA CPE was used in an anchored PCR strategy to amplify cDNAs that contain this sequence (Frohman et al. 1988; Loh et al. 1989). A degeneracy was introduced into the primer based on a comparison of putative CPE sequences from Xenopus, Spisula, Drosophila, and mouse. Several precautions were taken to increase PCR fidelity with the CPE primer, in light its of short size and AT-richness /see Materials and methods).

To date, 20 distinct clones, ranging from 12 to 145 nucleotides [excluding the poly(A) tail], have been isolated and sequenced. Seven clones contain the canonical polyadenylation signal AATAAA, suggesting that they encode $3^{\prime}$ ends of mRNAs. The other 13 clones lack this sequence and could represent the $3^{\prime}$ ends of mRNAs containing cryptic nuclear polyadenylation signals. Three of these non-AATAAA-containing clones have long poly(dA) stretches at their $3^{\prime}$ terminus, ranging from 40 to $>100$ nucleotides, suggesting that they encode $3^{\prime}$ ends of polyadenylated mRNAs. The validity of this approach for identifying potential stored maternal mRNAs was documented by isolation of the 3 '-terminal fragment of t-PA, a known stored maternal message (Huarte et al. 19871 .

\section{Analysis of extended $c D N A$ clones demonstrates the presence of CPE homologous sequences in $\mathrm{OM}-1$ and $19 \mathrm{G}$}

We initially focused our attention on two of these cDNA clones, termed OM-1 (for oocyte maturation) and 19G. These mRNAs are also present in F9 teratocarcinoma stem cells (undifferentiated embryonic mouse cells), allowing the isolation of cDNAs from a conventional F9 library. In F9 cells, mRNAs of $\sim 6.0 \mathrm{~kb}$ for OM-1 (see Fig. 7, below) and $3.6 \mathrm{~kb}$ for $19 \mathrm{G}$ (data not shown) are detected by Northern analysis.

Using complementary hybridization probes derived from the CPE clones, partial cDNAs for OM-1 and 19G were isolated from an F9 stem cell library. Sequence analysis of the F9 clones revealed that the extreme $3^{\prime}$ ends were identical to their respective CPE-derived clones. The analysis also revealed the presence of multiple regions homologous to the CPE /Fig. 3, stippled boxes), as are present in the $3^{\prime}$ sequences of other similarly regulated murine mRNAs (t-PA, Rickles et al. 1988; HPRT, Konecki et al. 1982). For both OM-1 and 19G, a CPE-like sequence in the F9 clone constitutes the 5' terminus of the clone derived with the CPE-PCR method and shows excellent homology to the CPE primer used (Fig. 3, underlined box; 12 of 12 nucleotides for OM-1; 11 of 12 nucleotides for 19G). Furthermore, the homologies extend both $5^{\prime}$ and $3^{\prime}$ of the core CPE element when compared with t-PA (Fig. 3). These results demonstrate
Figure 2. Approach used for isolating stored murine maternal mRNAs. Oocyte RNAs were reverse transcribed with an oligo(dT) primer/adapter. First-strand cDNAs were then amplified in the PCR using the oligo(dT) primer/adapter and the degenerate CPE primer. PCR-amplified products were then cloned for analysis. Hatched and stippled boxes represent adapter regions. Oligo(dT) primer/ adapteŕ, 5'-GCGAGCTCCGCGGCCGCG$\mathrm{T}_{(12) ;}$ CPE primer/adapter, 5'-TATGGTACCACTCGAGATTATGATTTT(A/T)AT.

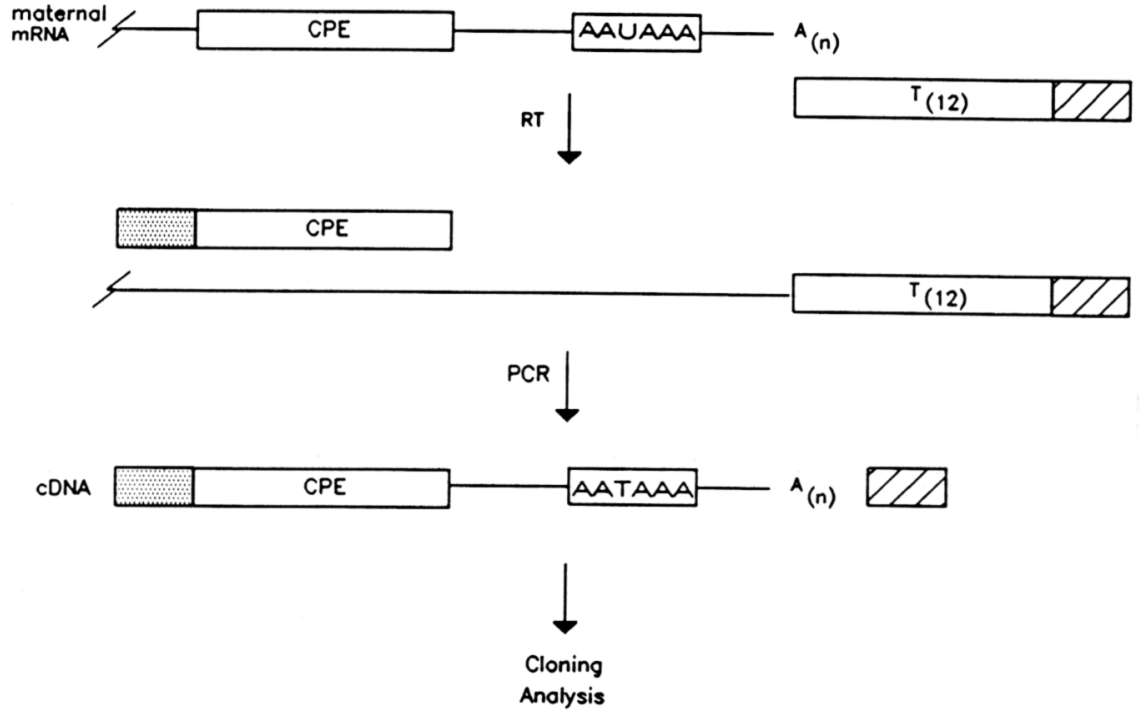


OM-1

TGCATGCCCCCCAGCAGTTAGGAGGGCAAGATTGCATTGAAGGGGTTTCAGACCTCTGAA GTAGTCAGTITTCTCCCATGTCTTCTATGGITTAATTGTAACATAGGTAGTATGCCTTCA CACTGGATCCCGGGGGCATGGGTITTGGAGATGTTGITITGTGGTGAATTGTCATCTGCA GTATTAAAGGGAAGACATATTCCTGCACATCACCATACCTTGTIITIAAAGCCAGAGTCA TTGTATGATITTITAITACCAGAAGTGCTAACCGGAATGTGATCTGGTTACCTTGGAAAT $\dot{ }$ tgtatgattttaataa (t-PA)

CAGGAACTTITATGAATGTACTGTAAAATAAAATGTGGGCTAATTCCCAG

196

CTCTACGTtGigCACAGAGAGCAGCCAGTITGAGTTITTAATGTAGGGCTGTGCCTTTGi

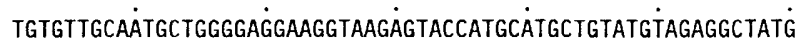
TCCTTACTGTGTGGAGCATTGTGTGCTTGGTATAACTGGCAGCCGTACATCCTTTACTGi

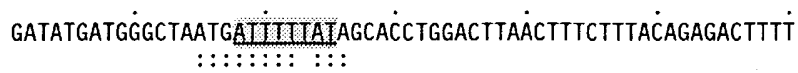
tgtatgatttiataa ( $t-P A)$

TCCCATAACTATTATTAGAÁCAAAGATATGCTTGAAAAATTATTAAATCÄAGTTGCCTTC

TGITTTTTCCंTAATATGTAAAACATTTTCC.TA $\overline{\text { AATAAA }}$ TGATAGAAAAGi

Figure 3. Sequence of $3^{\prime}$ end of OM-1 and $19 \mathrm{G}$ derived from F9 stem cell clones. CPE homologous sequences are shown in stippled boxes. Site of CPE primer annealing in the original CPE clone is underlined and compared with t-PA sequences (lowercase) from nucleotide 2373 to 2388 (see Fig. 1). Putative polyadenylation signals are both under- and overlined. Arrows denote start sites of synthetic transcripts for microinjection elongation assay (see Fig. 6). RsaI cleavage site is shown for OM-1 (see Fig. $5)$.

the ability of the primer to find its complement under the PCR conditions used.

The predicted amino acid sequence of a $3.2-\mathrm{kb}$ partial cDNA for OM-1 isolated from the F9 stem cell library (data not shown) shows no significant homology to previously identified proteins. A large translational reading frame of 295 amino acids, however, contains an acid-rich region (39\% Asp or Glu over 70 amino acids), the significance of which is unknown.

PCR test for the polyadenylation state of OM-1 and 19G

Because many mRNAs are present in low abundance and because it is difficult to obtain large numbers of mouse oocytes, conventional Northern analysis is unsatisfactory for routinely determining the poly(A) status of messages during maturation. Therefore, we have developed a PCR-based assay to determine whether an mRNA is regulated by polyadenylation upon meiotic maturation (Fig. 4). This assay is an application of anchored PCR (Frohman et al. 1988; Loh et al. 1989) and can rapidly determine the polyadenylation state of an mRNA in a single oocyte.

RNA was isolated from either primary or secondary oocytes and reverse transcribed with an oligo(dT) primer/adapter. In theory, the oligo(dT) primer/adapter can anneal anywhere on the poly(A) tail of the mRNA during the reverse transcription. For each specific mRNA, this procedure should generate cDNAs of variable heterogeneity in primary oocytes depending on the length of the poly(A) tail. If the specific mRNA is further polyadenylated upon meiotic maturation, an increase in the size and heterogeneity of the cDNAs should occur, resulting from the greater number of priming sites for the oligo(dT) primer/adapter. The resultant cDNAs were amplified with the oligo(dT) primer/adapter and a second sense primer that recognizes the specific clone. The adapter anchors the oligo(dT) primer preventing internal priming in the poly(dA) region, which would progressively shorten the PCR products. Increased heterogeneity can be visualized as a slower electrophoretic mobility of some of the PCR fragments. If the mRNA was not adenylated further, there should be no change in mobility of the amplified products.

As an initial control, the assay was performed for t-PA mRNA. As predicted, the mRNA with a short poly(A) tail (from primary oocytes) gave a compact set of products, whereas the form with a long poly(A) tail (from secondary oocytes) yielded a smear of products ranging up to several hundred nucleotides longer than those derived from the primary oocytes (Fig. 4). The PCR poly(A) test was then applied to OM-1 and 19G. OM-1 PCR products also show slower mobility upon maturation of the oocyte (Fig. 5, OM-1 $\mathrm{O}_{\mathrm{I}}$ vs. $\mathrm{O}_{\mathrm{II}}$ ), as seen with t-PA. Conversely, 19G PCR products do not change in size (Fig. 5, $19 \mathrm{G} \mathrm{O}_{\mathrm{I}}$ vs. $\mathrm{O}_{\mathrm{II}}$ ). To rule out the possibility that the heterogeneity was caused by spurious priming of the $5^{\prime}$ specific primer, the OM-1 PCR products were digested with the restriction endonuclease RsaI. This enzyme cleaves OM-1 31 nucleotides upstream of the poly(A) tail. Therefore, digestion of these PCR products from the different stages analyzed should yield a constant $5^{\prime}$ fragment of 65 nucleotides, as well as a heterogeneous $3^{\prime}$ end. Analysis of the restriction digest shows that the $5^{\prime}$ fragment is identical in size for both $\mathrm{O}_{\mathrm{I}}$ and $\mathrm{O}_{\mathrm{II}}$ (Fig. 5). The $3^{\prime}$ fragment, however, is retarded in its mobility upon maturation.

The PCR reaction under the conditions used is in the linear range of amplification (data not shown). Therefore, the data suggest that in primary oocytes OM-1 and 19G mRNAs are present at $\sim 10 \%$ the level seen for $t$-PA and that their levels decrease after meiotic maturation. By densitometric scanning of Figure 5, OM-1 decreases by $70 \%$ and $19 \mathrm{G}$ by $90 \%$; however, these observed de- 
Figure 4. PCR poly(A) test. (Left) Schematic representation of the test. If an mRNA is polyadenylated during maturation, the products from an anchored PCR amplification using a message-specific primer and oligo $\{\mathrm{dT}\rangle$ primer/adapter will become larger and more heterogeneous. (Right) Autoradiograph of the test applied to $t$-PA mRNA from primary $\left[\mid O_{\mathrm{U}}, \mathrm{t}\right.$-PA mRNA with short poly $(A)$ tail $]$ and secondary $\left[\mathrm{O}_{\mathrm{I}}, \mathrm{t}\right.$-PA mRNA with long poly(A) tail] oocytes. PCR was performed on one oocyte-equivalent of cDNA, and $5 \%\left(\mathrm{O}_{\mathrm{I}}\right)$ or $50 \%\left(\mathrm{O}_{\mathrm{II}}\right)$ of this sample was then analyzed by PAGE. The no-template control was negative (data not shown). t-PA sense oligonucleotide (nucleotides 2000-2025, Rickles et al. 1988), 5'-TCAGATGAGATGACAGGGAGATGCC; see Fig. 2 for oligo(dT) primer/adapter sequence.
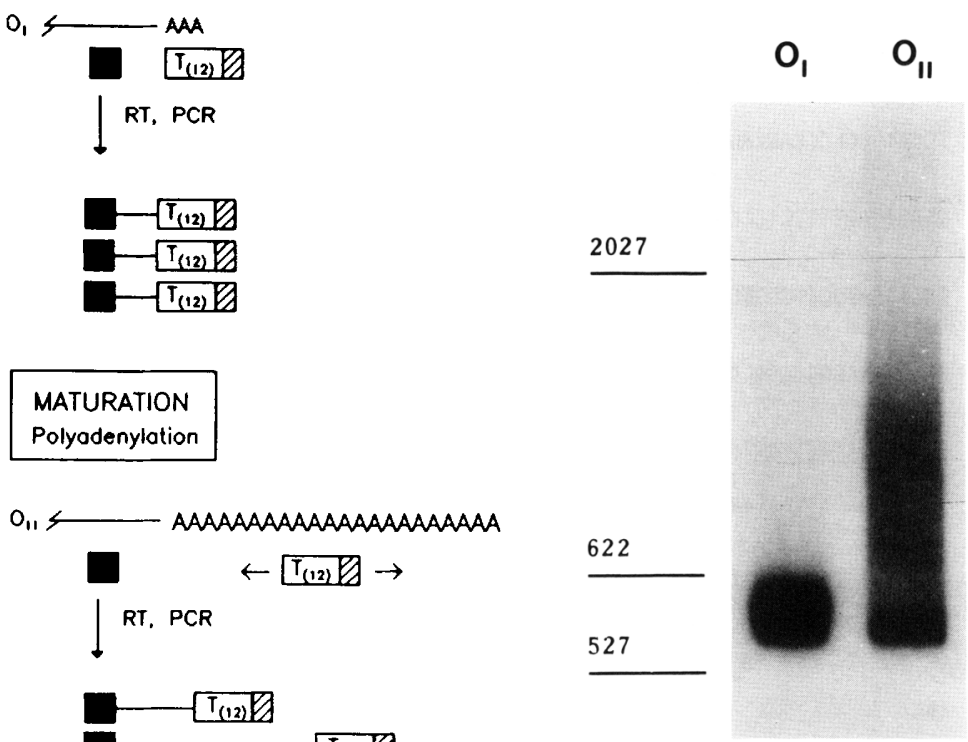

MATURATION

Polyodenylation

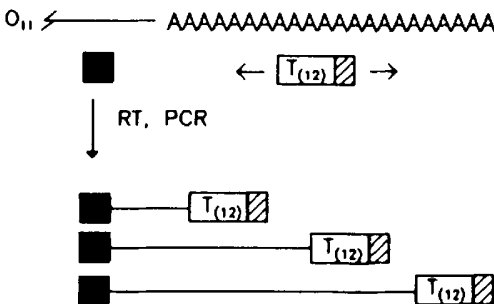

622

$$
527
$$

otides of OM-1 or 350 nucleotides of 19G (Fig. 3, arrows). OM-1 transcripts were elongated upon maturation as determined by a slower mobility of the isolated injected RNAs in secondary oocytes. 19G transcripts displayed no change in mobility (Fig. 6). Thus, these data agree with those obtained from the PCR poly(A) test.

\section{Northern analysis of OM-1 mRNA}

To establish directly that OM-1 mRNA is elongated upon oocyte maturation, Northern analysis using a specific OM-1 probe was performed (Fig. 7). A single species at $6.0 \mathrm{~kb}$ could be detected in $\mathrm{O}_{\mathrm{I}}$ RNA. This species exhibited slower mobility in $\mathrm{O}_{\mathrm{II}}(\sim 6.4 \mathrm{~kb})$, suggesting that the RNA had been polyadenylated. In F9, granulosa, and cumulus cells, a second band of equal or greater intensity at $4.5 \mathrm{~kb}$ could be detected. The significance of the different-sized transcripts is not known. These results, however, demonstrate that the signal detected in the oocyte samples is not the result of granulosa or cumulus cell contamination. This membrane was rehybridized with a probe recognizing the $18 \mathrm{~S}$ ribosomal subunit. All ribosomal bands had migrated to equal positions (data not shown).

\section{Antisense oligonucleotide microinjection destroys the $3^{\prime}$ end of OM-1 but does not inhibit the first meiotic division}

The cytoplasmic polyadenylation of OM-1 mRNA during oocyte maturation is likely to result in translational recruitment. Microinjection of antisense oligonucleotides has been shown to inhibit the translation of specific stored maternal messages in both Xenopus (Smith et al. 1990) and mouse oocytes (Strickland et al. 1988; 
$O M-1$

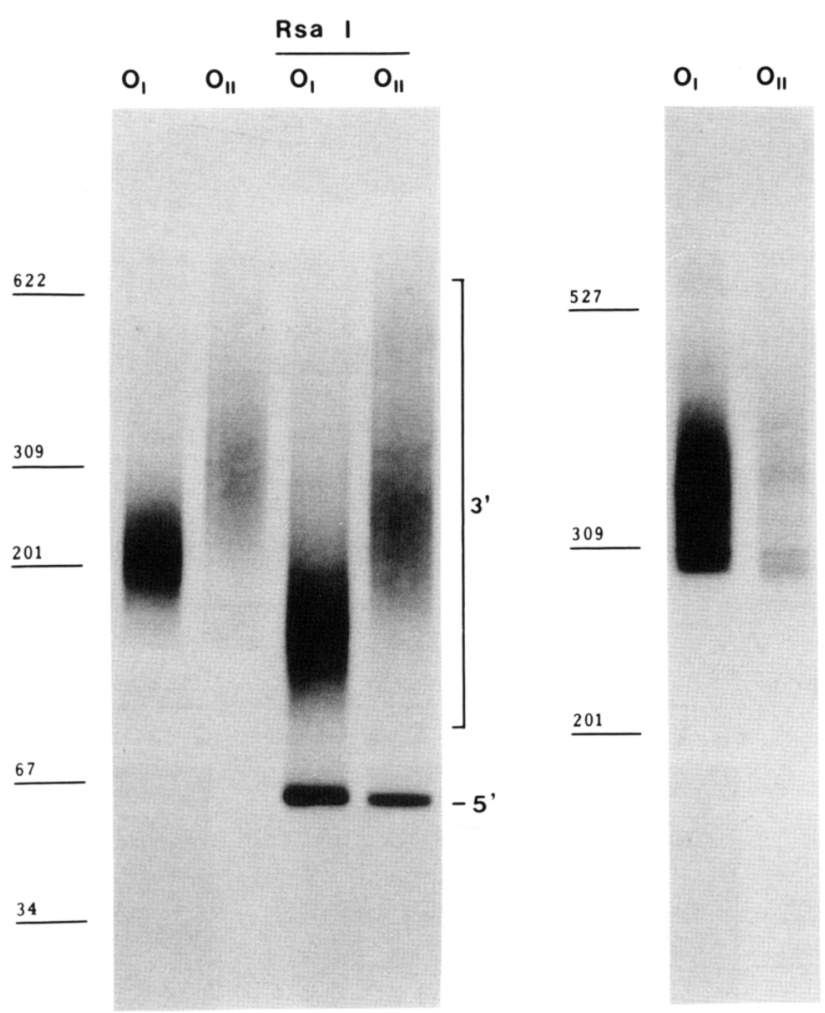

Figure 5. PCR poly(A) test of OM-1 (left) and 19G (right). Autoradiograph of PCR-amplified products demonstrating polyadenylation of OM-1 upon maturation. PCR-amplified products from lanes $\mathrm{O}_{\mathrm{I}}$ and $\mathrm{O}_{\mathrm{II}}(\mathrm{OM}-1)$ were digested with $R s a \mathrm{I}$ (see Fig. 3 for location of site) and electrophoresed, generating 5 ' fragments of equal size in both samples $\left(5^{\prime}\right)$. The $3^{\prime}$ fragments are of heterogeneous length and are bracketed $\left(3^{\prime}\right) .\left(\mathrm{O}_{\mathrm{I}}\right)$ Primary oocytes; $\left\langle\mathrm{O}_{\mathrm{II}}\right)$ secondary oocytes. OM-1 $\mathrm{O}_{\mathrm{I}}$ and $\mathrm{O}_{\mathrm{II}}$ lanes contain 0.25 oocytes of PCR-amplified material per lane; all other lanes contain 0.5 oocytes per lane. The no-template controls were negative in all cases (data not shown). OM-1 sense primer, 5'-ACCAGAAGTGCTAACCGGAATGTGA; 19G sense primer, 5'-GTACCATGCATGCTGTATGTAGAGG; see Fig. 2 for oligo(dT) primer/adapter sequence.

O'Keefe et al. 1989; Paules et al. 1989) and can be an effective means to analyze their function.

To determine whether OM-1 mRNA is required for oocyte maturation, antisense oligodeoxynucleotides directed against the message were injected into primary oocytes. These injections had no visible effect on meiotic maturation, as evidenced by polar body extrusion. PCR analysis was used to determine whether OM-1 mRNA had been degraded (Fig. 8). Electrophoretic analysis of the PCR-amplified products demonstrated that the regions of OM-1 mRNA downstream of the site of oligonucleotide hybridization were destroyed in the secondary oocyte. This was evidenced by a significant decrease in cDNAs derived from antisense-injected oocytes but not from the sense-injected controls. Antisense oligodeoxynucleotides directed against $t-P A$ mRNA also led to instability of the message downstream of the duplex region (data not shown). The OM-1 and t-PA results in this assay suggest that as in antisense RNA injections for t-PA (Strickland et al. 1988), the 5' portion of the shortened message remains stable even after maturation when the unaltered message is normally degraded. The remaining $5^{\prime}$ portion should not be translationally regulated because it lacks both the CPE and AAUAAA.

\section{Proportion of CPE-derived cDNA clones representing maternal $m R N A$ s that are regulated by cytoplasmic polyadenylation}

Given the AT-richness and short length of the CPE primer, it was not surprising that presumably irrelevant clones were isolated. For example, one clone has $>90 \%$ homology to a $5^{\prime}$ fragment of the $\beta$-subunit of human translation initiation factor 2 (TIF2). This clone may represent the murine homolog and indicates that background clones were also isolated. With the exception of t-PA and TIF2, none of the clones show significant homology to sequences in either the EMBL or GenBank data bases.

Table 1 summarizes the seven cDNA clones containing AATAAA that have been isolated and partially characterized. By use of the PCR poly(A) test, three of these clones appear to be polyadenylated upon meiotic maturation: $16 \mathrm{~A}(\mathrm{t}-\mathrm{PA}), \mathrm{OM}-1$, and $\mathrm{OM}-2$. Therefore, at least these 3 of the 20 clones isolated to date $(15 \%)$ appear to be regulated by cytoplasmic polyadenylation.

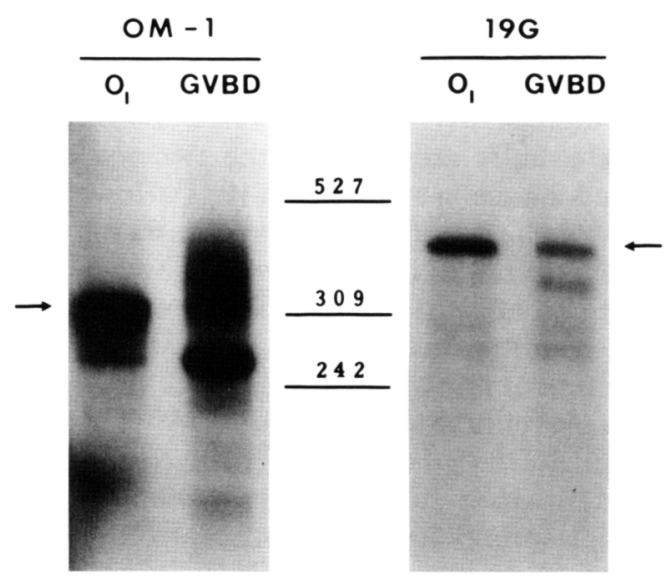

Figure 6. Elongation of microinjected radiolabeled RNA transcripts. Autoradiographs of oocyte RNA extracts after $6 \%$ denaturing PAGE for both OM-1 (left) and 19G (right). Oocytes were cultured for $16 \mathrm{hr}$ after microinjection. $\left(\mathrm{O}_{\mathrm{I}}\right)$ Primary oocytes cultured in the presence of cAMP; (GVBD) Germinal vesicle breakdown stage (meiotically maturing) oocytes. The arrows show the sizes of the injected RNAs. The appearance of the lower species in both OM-1 and 19G GVBD lanes is presumably the result of degradation of the transcripts upon maturation, as is seen with $t-P A$ (Vassalli et al. 1989). OM-1, $\mathrm{O}_{\mathrm{I}}$ and GVBD, respectively, have 12 and 16 oocytes per lane; 19G has 10 oocytes per lane. 


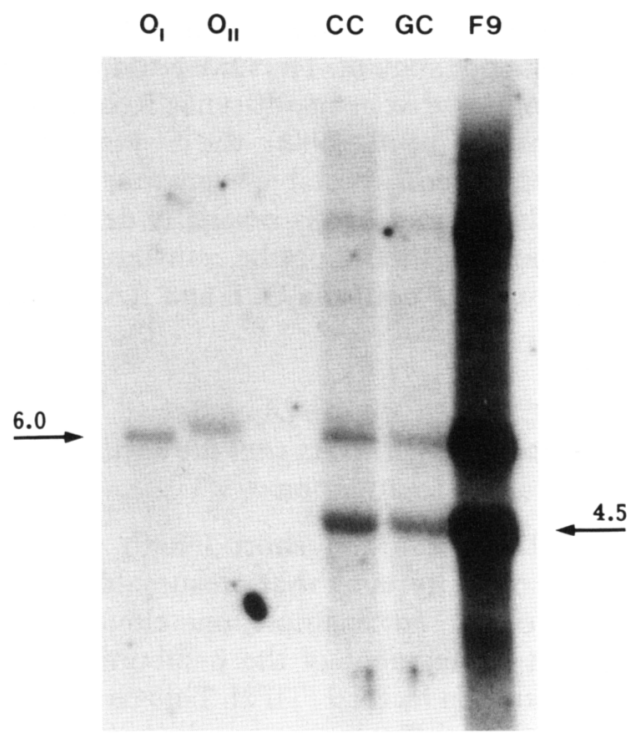

Figure 7. Northern analysis of OM-1 mRNA. RNA preparations from 265 primary oocytes $\left(\mathrm{O}_{\mathrm{I}}\right), 265$ secondary oocytes $\left(\mathrm{O}_{\mathrm{II}}\right)$, $2 \times 10^{5}$ cumulus cells that surround secondary oocytes after ovulation $(\mathrm{CC}), 2 \times 10^{5}$ granulosa cells from preovulatory ovarian follicles $(\mathrm{GC})$, and $5 \mu \mathrm{g}$ of F9 stem cell total RNA were probed with a 2.3-kb antisense riboprobe directed against OM-1. The sizes of the hybridizing species were estimated from another gel that included molecular size markers. The increase in size of the $\mathrm{O}_{\mathrm{II}}$ mRNA was estimated to be between 200 and 400 nucleotides.

\section{Discussion}

\section{Isolation of translationally regulated maternal mRNAs from mouse oocytes}

In mouse, there is little or no transcription from the time the oocyte is fully grown until the two-cell stage (for review, see Magnuson and Epstein 1981). However, there are extensive changes in protein synthesis during these stages (Golbus and Stein 1976; Schultz and Wassarman 1977a; Latham et al. 1991). The changes are dictated in part by translational control, as illustrated by the fact that inhibitors of protein synthesis block these early developmental events (Golbus and Stein 1976; Schultz and Wassarman 1977b; Howlett 1986), whereas inhibitors of RNA synthesis have little effect (Levey et al. 1977). Because cytoplasmic polyadenylation controls the translational recruitment of many dormant mRNAs, it is likely that these elongated mRNAs are responsible, in large part, for early developmental control.

We report the isolation of cDNA clones representing mRNAs that are regulated by cytoplasmic polyadenylation upon meiotic maturation. These cDNAs are small fragments of the $3^{\prime}$ regions of mRNAs and provide sufficient information to perform the PCR poly(A) test and functional analysis via antisense oligonucleotide injection. The clones can also be used as probes for the isolation of full-length cDNAs from other libraries, or extended sequence in the $5^{\prime}$ direction can be obtained using

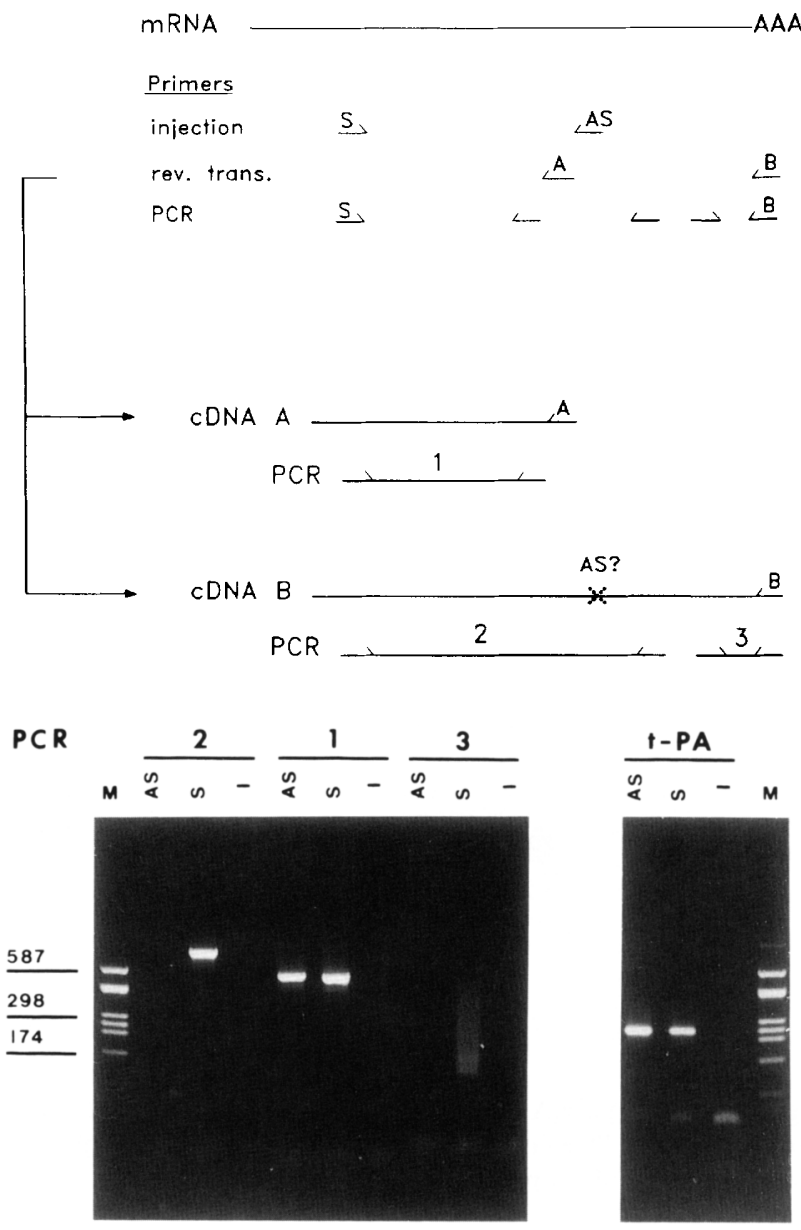

Figure 8. Antisense oligonucleotide injection destroys the $3^{\prime}$ end of OM-1 mRNA in the oocyte. Oocytes were microinjected with either a sense (S) or an antisense (AS) oligonucleotide and cultured overnight to allow maturation. Isolated RNA from fully mature secondary oocytes was reverse transcribed with either primer A (5'-AAATGAGAGATGGCAAAATACAGGG) or B [oligo(dT) primer/adapter; see Fig. 2]. PCR was then used to amplify products 1,2 , or 3 as shown. Locations of the primers are accurate with respect to one another. (Bottom) Ethidium bromide-stained $2 \%$ agarose gel of PCR-amplified products. The PCR number refers to the product number from the top. (AS\} Antisense-injected oocyte; (S) sense-injected oocyte; (-) no template control; (M) markers (pUC9-HaeIII digest); (t-PA) amplification of $t$-PA sequences from OM-1-microinjected oocytes reverse-transcribed with primer $B$, suggests that the oligonucleotide has no nonspecific effects on other mRNAs. The absence of a strong signal for both products 2 and 3 in the AS lanes suggests that the $3^{\prime}$ end of the message has been destroyed. The presence of a signal in product 1 AS suggests that the $5^{\prime}$ portion of the mRNA is stable. Appropriate signal is seen in all S-injected lanes. The smear seen for product 3 in the $S$ lane is caused by the poly $(\mathrm{A})$ tail on the message [PCR poly(A) test, see Fig. 5]. Injection primers: (AS) 5'-TGCAATCTTGCCCTTCTAACTGCTG; (S) 5'-TTGGCCTCAAGATTGCAGTCCTGAC. PCR products: (1) 3' AS primer, 5'-ATCCTGCCAGTTGCTGATGG; (2) 3' AS primer, 5'-GACATGGGAGAAAACTGAC$\mathrm{TACTTC}_{i}(3) 5^{\prime} \mathrm{S}$ primer, see Fig. 5 OM1 sense primer. $t$-PA primers: 5' S primer, see Fig. 4 t-PA sense oligonucleotide; $3^{\prime}$ AS primer (nucleotides 2230-2255, Rickles et al. 1988), 5'-TATGGTGCTGTGATTCGGCCAGATA. 
Table 1. Summary of AATAAA-containing CDNA clones isolated by CPE primer-based PCR

\begin{tabular}{lcc}
\hline Clone & Size (nucleotides) & PCR poly(A) test \\
\hline 16A (t-PA) & 118 & $+^{\mathrm{a}}$ \\
OM-1 & 95 & $+^{\mathrm{a}}$ \\
19G & 145 & $-{ }^{\mathrm{b}}$ \\
OM-2 & 65 & + \\
15D & 71 & - \\
14A & 67 & - \\
15C & 72 & -
\end{tabular}

Listing of the seven AATAAA-containing cDNA clones showing the size between the CPE primer sequence and the start of the poly(dA) stretch. The results of the PCR poly(A) test are shown as cytoplasmically polyadenylated upon meiotic maturation $(+)$ or not further polyadenylated at maturation $(-1$. Clone 16A encodes the $3^{\prime}$ end of the t-PA mRNA.

${ }^{a}$ Cytoplasmic polyadenylation confirmed by microinjection of RNA fragments and by Northern analysis ( $t-P A$, Huarte et al. 1987, Vassalli et al. 1989: OM-1, this paper). Multiple isolates of both of these clones were obtained.

bLack of cytoplasmic polyadenylation upon maturation confirmed by microinjection of RNA fragments.

PCR protocols (Frohman et al. 1988). The strategy described is applicable for isolating mRNAs that are polyadenylated at other times during early embryogenesis.

The cDNA clones were isolated by utilizing a conserved motif that appears to be required for cytoplasmic polyadenylation. This approach has been validated by three main pieces of evidence. First, multiple clones encoding the $3^{\prime}$ UTR of t-PA mRNA, a stored maternal message that is regulated by the cytoplasmic polyadenylation element, were obtained (Table 1). Second, analysis of clones OM-1 and 19G, reisolated from a conventional F9 stem cell cDNA library, confirmed the presence of sequences with homology to the CPE that were accurately targeted by the AT-rich degenerate CPE primer in the PCR (Fig. 3). Third, and most importantly, two novel mRNAs, OM-1 and OM-2, which are polyadenylated upon maturation, were isolated.

Because there are divergent sequences that are functionally similar to the CPE, such as UUUUAAAU in mouse HPRT (McGrew et al. 1989), the conditions used in the PCR may have been too stringent to clone all cytoplasmically polyadenylated mRNAs. Therefore, primers with greater degeneracies can be used under less stringent conditions to help identify other potentially interesting mRNAs.

\section{Analysis of OM-1 and 19G}

Using a novel PCR poly(A) test, a microinjection assay, and conventional Northern analysis, we have shown that $\mathrm{OM}-1$ is elongated at its $3^{\prime}$ end upon meiotic maturation. Because priming and amplification occur via oligo(dT), the shift in size of OM-1 PCR products after maturation (Fig. 5) indicates that this modification is caused by cytoplasmic polyadenylation as observed with other stored maternal mRNAs (Rosenthal et al. 1983; Dworkin and Dworkin-Rastl 1985; Huarte et al. 1987; Paynton et al. 1988; McGrew et al. 1989). Transcripts derived from clone 19G are not elongated upon injection, and the mRNA does not show reduced mobility in the PCR poly(A) test upon maturation. The lack of a mobility shift in the in vivo elongation assay could be explained if the RNA fragment lacked sequences required for proper regulation, because only a portion of the $3^{\prime}$ UTR was injected. However, the data derived from the PCR poly(A) test, a method that analyzes the endogenous message, suggest that the mRNA is not elongated.

OM-1 mRNA appears to be polyadenylated to a greater extent than the dormant $t-P A$ mRNA in primary oocytes. First, the presence of a relatively long poly(A) tail could allow translation of the OM-1 message in primary oocytes; and, upon maturation, further polyadenylation would lead to continued or increased translation. This strategy could bypass the default deadenylation and translational inactivation that is seen with mRNAs that are translated in the primary oocyte (Fox and Wickens 1990; Varnum and Wormington 1990). Alternatively, the length of poly(A) tail in primary oocytes may not be sufficient to allow translation of the message, with further polyadenylation needed for initiation, as has been shown for Xenopus G-10 mRNA (McGrew et al. 1989).

One difference in the poly(A) test PCR products observed for OM-1 and t-PA is notable (cf. Figs. 4 and 5). With t-PA, a discrete lower boundary is visible in both primary and secondary oocytes, as expected, because the poly(A) tail begins at a defined position. However, with OM-1 no clearly defined lower boundary is detected. On the basis of primary sequence, the smallest 3' fragment of OM-1 in RsaI-digested samples (Fig. 5) should be 61 nucleotides (31 nucleotides of OM-1 sequences and 30 nucleotides of primer sequence). Instead, the smallest fragment is larger. This pattern is consistent with preferential priming at an internal region of the poly(A) tail that is more accessible as a result of secondary structure during the reverse transcription.

The fact that 19G contains three CPE-like sequences is intriguing in light of its lack of further polyadenylation during oocyte maturation (Fig. 5) or fertilization (data not shown). One possible explanation is that 19G could be regulated by polyadenylation at a subsequent developmental stage. In this case, stable 19G mRNA would be deadenylated upon maturation and then readenylated at some other point in the early development of the embryo. Multiple changes in the polyadenylation state of Xenopus Xfin mRNA have been reported (Ruiz i Altaba et al. 1987). A second possibility is that the CPE sequences in 19G are located too far upstream of the polyadenylation signal to be functional.

If $19 \mathrm{G}$ mRNA is not adenylated during development, it implies that having both the CPE sequence and an AAUAAA is not sufficient for cytoplasmic polyadenylation, but that other structural features are required for poly(A) elongation. A role for the sequence context of the CPE is also suggested by the inability to polyadenylate all transcripts that contain four copies of the sequence in an irrelevant RNA (Fig. 1C). 
Microinjection of antisense oligonucleotides
to identify the function of maternal mRNAs

Selectively isolating cDNAs and establishing an effective method to screen the candidate clones are the first two steps in analyzing maternal mRNAs. The third step, determining the function of the transcripts, can be addressed by antisense DNA microinjection. The oocyte is ideally suited for antisense technology, because there is little or no transcription upon the resumption of meiosis through the first zygotic cleavage in mouse (Flach et al. 1982; Pikó and Clegg 1982). Thus, antisense-mediated destruction of an mRNA can be used to generate a transient loss-of-function phenotype and reveal its effect on early developmental processes. Antisense oligodeoxynucleotides and RNA transcripts have been used extensively to study the role of maternal mRNAs in these early events. With c-mos and c-ets, antisense DNA injections have been shown to disrupt meiosis (Sagata et al. 1988; O'Keefe et al. 1989; Paules et al. 1989; Chen et al. 1990). Antisense oligonucleotide-mediated destruction of RNAs is believed to occur in the duplexed region via an RNase activity (Strickland et al. 1988). If the duplexed region lies upstream of either the CPE or AAUAAA, the mRNA cannot be polyadenylated, and translation is, therefore, prevented.

Antisense oligodeoxynucleotides directed against either OM-1 or 19G fail to block meiotic maturation of the oocyte. Destruction of the mRNA in the region targeted by the antisense DNA was confirmed for OM-1 by PCR analysis (Fig. 8). If the translational regulation of other stored maternal mRNAs serves as a model, this truncation should disrupt translation (Sagata et al. 1988; Strickland et al. 1988). The lack of effect by this disruption could have several explanations, for example, (1) the product of this message is required at a later time point, such as fertilization; (2) oocyte maturation is rescued by a redundant factor; $(3)$ the mRNA is translated in primary oocytes, and the protein persists throughout maturation.

\section{Conclusions}

In Drosophila, genetic screens have allowed the isolation of maternal-effect genes that are required only for processes under maternal control (Nüsslein-Volhard et al. 1987). These screens do not identify another set of genes that are required both maternally and in stages controlled by the zygotic genome. The strategy described here identifies maternal mRNAs that are good candidates for regulators of early embryogenesis, irrespective of their function at other developmental stages. The use of antisense-mediated loss of function will allow concentration on mRNAs whose deficiency leads to particularly interesting phenotypes. Given the fact that oocytes contain an extraordinarily complex population of RNA (Davidson 1976), this combined approach should facilitate the otherwise daunting task of isolating maternal genes that direct early mouse development.

\section{Materials and methods}

General methods

The following procedures were used for oocytes (Sallés et al. 1992): collection (Huarte et al. 1985), culture (Poueymirou and Schultz 1989), injection (Strickland et al. 1988). Oligonucleotides were purchased from Operon Technologies, Inc. (Alameda, CA). Primers were not purified by high-performance liquid chromatography.

Construction of $t-P A$ deletion mutants and CPE-multimerized clones

Clone B4 containing 356 nucleotides of the 3' UTR of $t$-PA 99 nucleotides upstream of the polyadenylation site (Vassalli et al. 1989) was digested with PstI and EcoRI. The resultant 500-bp fragment was isolated and cloned into the cognate sites in pBS (Stratagene) yielding plasmid pDdSA. pDdSA was then linearized with $B a m H I$ and treated with Bal3l exonuclease. The reaction was stopped at various times, and the aliquots were pooled and ligated to BamHI linkers. The pooled sample was then digested with PstI and BamHI, and the fragments were isolated.

A second aliquot of pDdSA was digested with PstI and $B a m H I$, and the large fragment was gel isolated. The inserts from above were ligated to this vector. The resultant clones contain a common $5^{\prime} \mathrm{t}$-PA end point, a shortened $3^{\prime}$ end point, and a BamHI site followed by the AATAAA sequence.

For the CPE-multimerized clones, pDdSA was digested with $B a m H I$ and ligated to the annealed oligonucleotides $\left(S, 5^{\prime}\right.$ GATCTATGATTTTAATAATG; AS, 5'-GATCCATTATTAAAATCATA). This resulted in constructs containing polylinker sequences upstream of the oligomerized CPE sequences upstream of AATAAA. Clones containing one to four copies of the sequence in the proper orientation were used.

\section{Isolation of CPE-containing $C D N A$ clones}

RNA from primary oocytes was isolated (Schibler et al. 1980; Huarte et al. 1987) and reverse transcribed with 15 units of AMV reverse transcriptase (Life Sciences) (Rickles et al. 1988) using $200 \mathrm{ng}$ of the oligo(dT) primer/adapter. The GC-rich adapter region allows increased annealing temperature in the subsequent PCR. Two oocyte equivalents of first-strand cDNAs were then amplified using Amplitaq (U.S. Biochemical). All components were mixed together, except the primers. Immediately before amplification, $2.5 \mathrm{ng}$ of end-labeled CPE primer (sp. act. $2.8 \times 10^{8} \mathrm{cpm} / \mu \mathrm{g}$ ) was added. Samples were denatured for $5 \mathrm{~min}$ at $93^{\circ} \mathrm{C}$ and cycled twice with $30 \mathrm{sec}$ at $93^{\circ} \mathrm{C}, 1 \mathrm{~min}$ at $45^{\circ} \mathrm{C}$, and $1 \mathrm{~min}$ at $72^{\circ} \mathrm{C}$. The samples were then incubated at $93^{\circ} \mathrm{C}$ for $3 \mathrm{~min}$ at which time 20 pmoles of oligo(dT) primer/ adapter was added in a $1-\mu 1$ volume, followed by 30 cycles of 30 $\mathrm{sec}$ at $93^{\circ} \mathrm{C}, 1 \mathrm{~min}$ at $60^{\circ} \mathrm{C}, 1 \mathrm{~min}+2 \mathrm{sec} / \mathrm{cycle}$ at $72^{\circ} \mathrm{C}$, and a 7 -min final incubation at $72^{\circ} \mathrm{C}$. Only two cycles were carried out at the lower temperature, before adding additional oligo(dT) primer/adapter to minimize spurious priming of the AT-rich primers.

To decrease the selective amplification of abundant and smaller sequences, the initial PCR reaction was size fractionated by electrophoresis on an $8 \%$ nondenaturing acrylamide gel. The gel was dried and exposed to film. Slices ranging from 20 to $30 \mathrm{bp}$ of the visible smearing patterns on the autoradiograph were excised from the gel. Half of the gel slice was rehydrated in 50-100 $\mu \mathrm{l}$ of $\mathrm{H}_{2} \mathrm{O}$, the paper backing was removed, and the gel slice was crumbled by centrifugation through a small opening in a $500-\mu l$ Eppendorf tube into a $1.5-\mathrm{ml}$ Eppendorf tube. Fifty 
microliters of the crushed gel was added to $50 \mu \mathrm{l}$ of a $2 \times$ PCR cocktail containing 50 pmoles of each primer, denatured for 5 min at $93^{\circ} \mathrm{C}$, and amplified for 30 cycles under the following conditions: $30 \mathrm{sec}$ at $93^{\circ} \mathrm{C}, 1 \mathrm{~min}$ at $60^{\circ} \mathrm{C}$, and $1 \mathrm{~min}$ at $72^{\circ} \mathrm{C}$. After cycling, samples were incubated at $72^{\circ} \mathrm{C}$ for $7 \mathrm{~min}$. This size-fractionation and reamplification process served to decrease the number and heterogeneity of individual clones in each sample.

The reamplified DNA was digested with both NotI and XhoI. Linker fragments were removed by centrifugation of the digest through an A-5M (Bio-Rad) spin column (Sambrook et al. 1989). The flowthrough was ligated to pKS + digested with the same enzymes. The ligation reaction was then electroporated into XL1-S, a derivative of XL-1 blue (Stratagene) which gave 100fold greater electrotransformation efficiencies $1>10^{10}$ transformants/ $\mu \mathrm{g}$ DNA) using a GenePulser (Bio-Rad). Clones were sequenced by Sequenase (U.S. Biochemical) using PEG-precipitated miniprep DNA (Sambrook et al. 1989).

\section{Northern analysis, transcript synthesis, and isolation of recombinant $\lambda$ phage $c D N A$ clones from $F 9$ stem cell library}

Northern analysis was performed (Huarte et al. 1987) with Duralon membranes (Stratagene). Transcripts were prepared as described (Strickland et al. 1988). Clones for OM-1 and 19G were isolated from an F9 stem cell $\lambda$ gtl0 library (gift of Dr. Barry Shur) using random-primed probes (Sambrook et al. 1989).

\section{PCR poly $(A)$ test}

Primary and secondary oocyte cDNAs were prepared (see above) and amplified in the presence of 25 pmoles each of a specific primer directed against the clone of interest, and the general oligo(dT) primer/adapter used for the cDNA synthesis in a 50- $\mu$ l reaction that contained $10 \mu \mathrm{Ci}$ of $\left[\alpha{ }^{32} \mathrm{P}\right] \mathrm{dATP}$ (sp. act. 3000 $\mathrm{Ci} / \mathrm{mmole}$ ) as follows: $5 \mathrm{~min}$ at $93^{\circ} \mathrm{C}$, then 27 cycles of $30 \mathrm{sec}$ at $93^{\circ} \mathrm{C}, 1 \mathrm{~min}$ at $60^{\circ} \mathrm{C}$, and $1 \mathrm{~min}+5 \mathrm{sec} / \mathrm{cycle}$ at $72^{\circ} \mathrm{C}$. Cycling was followed by a $7-\mathrm{min}$ incubation at $72^{\circ} \mathrm{C}$. tRNA $(15 \mu \mathrm{g})$ was then added; samples were phenol/chloroform-extracted and precipitated with 0.5 volume $7.5 \mathrm{M}$ ammonium acetate and 2 volumes of ethanol. Products were electrophoresed on a $5 \%$ nondenaturing polyacrylamide gel.

\section{Acknowledgments}

We are very grateful to Drs. JoAnne Engebrecht, Peter Gergen, Joaquin Huarte, Richard Rickles, Richard Schultz, Jean-Dominique Vassalli, and members of our laboratory for stimulating discussions and helpful comments. We also thank Toni Daraio for artwork. This work was supported by grants HD-25922 and HD-17875 from the National Institutes of Health.

The publication costs of this article were defrayed in part by payment of page charges. This article must therefore be hereby marked "advertisement" in accordance with 18 USC section 1734 solely to indicate this fact.

\section{References}

Bachvarova, R., V. DeLeon, A. Johnson, G. Kaplan, and B.V. Paynton. 1985. Changes in total RNA, polyadenylated RNA, and actin mRNA during meiotic maturation of mouse oocytes. Dev. Biol. 108: 325-331.

Chen, Z.-Q., L.A. Burdett, A.K. Seth, J.A. Lautenberger, and T.S. Papas. 1990. Requirement of ets-2 expression for Xenopus oocyte maturation. Science 250: 1416-1418.

Davidson, E.H. 1976. Gene activity in early development, 2nd ed. Academic Press, New York.

Dworkin, M.B. and E. Dworkin-Rastl. 1985. Changes in RNA titers and polyadenylation during oogenesis and oocyte maturation in Xenopus laevis. Dev. Biol. 112: 451-457.

- 1990 . Functions of maternal mRNA in early development. Mol. Reprod. Dev. 26: 261-297.

Flach, G., M.H. Johnson, P.R. Braude, R.A.S. Taylor, and V.N. Bolton. 1982. The transition from maternal to embryonic control in the 2-cell mouse embryo. EMBO I. 1: 681-686.

Fox, C.A. and M. Wickens. 1990. Poly(A) removal during oocyte maturation: A default reaction selectively prevented by specific sequences in the 3' UTR of certain maternal mRNAs. Genes \& Dev. 4: 2287-2298.

Fox, C.A., M.D. Sheets, and M.P. Wickens. 1989. Poly(A) addition during maturation of frog oocytes: Distinct nuclear and cytoplasmic activities and regulation by the sequence UUUUUAU. Genes \& Dev. 3: 2151-2162.

Frohman, M.A., M.K. Dush, and G.R. Martin. 1988. Rapid production of full-length cDNAs from rare transcripts: Amplification using a single gene-specific oligonucleotide primer. Proc. Natl. Acad. Sci. 85: 8998-9002.

Golbus, M.S. and M.P. Stein. 1976. Qualitative patterns of protein synthesis in the mouse oocyte. I. Exp. Zool. 198: 337342.

Howlett, S.K. 1986. A set of proteins showing cell cycle dependent modification in the early mouse embryo. Cell 45: 387396.

Huarte, J., D. Belin, and J.-D. Vassalli. 1985. Plasminogen activator in mouse and rat oocytes: Induction during meiotic maturation. Cell 43: 551-558.

Huarte, J., D. Belin, A. Vassalli, S. Strickland, and J.-D. Vassalli. 1987. Meiotic maturation of mouse oocytes triggers the translation and polyadenylation of dormant tissue-type plasminogen activator mRNA. Genes \& Dev. 1: 1201-1211.

Konecki, D.S., J. Brennand, J.C. Fuscoe, C.T. Caskey, and A.C. Chianault. 1982. Hypoxanthine-guanine phosphoribosyltransferase genes of mouse and chinese hamster: Construction and sequence analysis of cDNA recombinants. Nucleic Acids Res. 10: 6763-6775.

Latham, K.E., J.I. Garrels, C. Chang, and D. Solter. 1991. Quantitative analysis of protein synthesis in mouse oocytes: I. Extensive reprogramming at the one- and two-cell stages. Development 112: 921-932.

Levey, I.L., D.E. Troike, and R.L. Brinster. 1977. Effects of alphaamanitin on the development of mouse ova in culture. $J$. Reprod. Fertil. 50: 147-150.

Loh, E.Y., J.F. Elliott, S. Cwirla, L.L. Lanier, and M.M. Davis. 1989. Polymerase chain reaction with single-sided specificity: Analysis of T cell receptor $\delta$ chain. Science 243: 217220.

Magnuson, T. and C.J. Epstein. 1981. Genetic control of very early mammalian development. Biol. Rev. 56: 369-408.

McGrew, L.L. and J.D. Richter. 1990. Translational control by cytoplasmic polyadenylation during Xenopus oocyte maturation: Characterization of cis and trans elements and regulation by cyclin/MPF. EMBO J. 9: 3743-3751.

McGrew, L.L., E. Dworkin-Rastl, M.B. Dworkin, and J.D. Richter. 1989. Poly(A) elongation during Xenopus oocyte maturation is required for translation recruitment and is mediated by a short sequence element. Genes \& Dev. 3: 803-815.

Mutter, G.L., G.S. Grills, and D.J. Wolgemuth. 1988. Evidence for the involvement of the proto-oncogene c-mos in mammalian meiotic maturation and possibly very early development. EMBO I. 7: 683-689. 
Nüsslein-Volhard, C., H.G. Frohnhöfer, and R. Lehmann. 1987. Determination of anteroposterior polarity in Drosophila. Science 238: 1675-1681.

O'Connell, M. 1989. "Plasminogen activator regulation in granulosa cells and oocytes." Ph.D. thesis. State University of New York, Stony Brook, New York.

O'Keefe, S.J., H. Wofes, A.A. Kiessling, and G.M. Cooper. 1989. Microinjection of antisense c-mos oligonucleotide prevents meiosis II in the maturing mouse egg. Proc. Natl. Acad. Sci. 86: 7038-7042.

Paris, J. and J.D. Richter. 1990. Maturation-specific polyadenylation and translational control: Diversity of cytoplasmic polyadenylation elements, influence of poly(A) tail size, and formation of stable complexes. Mol. Cell Biol. 10: 56345645.

Paris, J., K. Swenson, H. Piwnica-Worms, and J.D. Richter. 1991. Maturation-specific polyadenylation: In vitro activation by $\mathrm{p} 34^{\mathrm{cdc2}}$ and phosphorylation of a $58-\mathrm{kD}$ CPE-binding protein. Genes \& Dev. 5: 1697-1707.

Paules, R.S., R. Buccione, R.C. Moschel, G.F. Vande Woude, and J.J. Eppig. 1989. Mouse Mos proto-oncogene product is present and functions during oogenesis. Proc. Natl. Acad. Sci. 86: 5395-5399.

Paynton, B.V., R. Rempel, and R. Bachvarova. 1988. Changes in state of adenylation and time course of degradation of maternal mRNAs during oocyte maturation and early embryonic development in the mouse. Dev. Biol. 129: 304-314.

Pikó, L. and K.B. Clegg. 1982. Quantitative changes in total RNA, total poly(A), and ribosomes in early mouse embryos. Dev. Biol. 89: 362-378.

Poueymirou, W.T. and R.M. Schultz. 1989. Regulation of mouse preimplantation development: Inhibition of synthesis of proteins in the two-cell embryo that require transcription by inhibitors of cAMP-dependent protein kinase. Dev. Biol. 133: 588-599.

Richter, J.D. 1991. Translational control during early development. BioEssays 13: 179-183.

Rickles, R.J., A.D. Darrow, and S. Strickland. 1988. Molecular cloning of complementary DNA to mouse tissue plasminogen activator mRNA and its expression during F9 teratocarcinoma cell differentiation. $/$. Biol. Chem. 263: 1563-1569.

Rosenthal, E.T. and F.H. Wilt. 1987. Selective messenger RNA translation in marine invertebrate oocytes, eggs, and zygotes. In Translational regulation of gene expression (ed. J. Ilan), pp. 87-110. Plenum Press, New York.

Rosenthal, E.T., T.R. Tansey, and J.V. Ruderman. 1983. Sequence-specific adenylations and deadenylations accompany changes in the translation of maternal messenger RNA after fertilization of Spisula oocytes. J. Mol. Biol. 166: 309-327.

Ruiz i Altaba, A., H. Perry-O'Keefe, and D.A. Melton. 1987. $X$ fin: an embryonic gene encoding a multifingered protein in Xenopus. EMBO J. 6: 3065-3070.

Sagata, N., M. Oskarsson, T. Copeland, J. Brumbaugh, and G.F. Vande Woude. 1988. Function of c-mos proto-oncogene product in meiotic maturation in Xenopus oocytes. Nature 335: 519-525.

Sallés, F.J., W.G. Richards, J. Huarte, J.-D. Vassalli, and S. Strickland. 1992. Microinjection of antisense sequences into primary mouse oocytes. Guide to techniques in mouse development. Methods Enzymol. (in press).

Sambrook, I., E.F. Fritsch, and T. Maniatis. 1989. Molecular cloning: A laboratory manual. 2nd ed. Cold Spring Harbor Laboratory Press, Cold Spring Harbor, New York.

Schibler, U., M. Tosi, A.-C. Pittet, L. Fabiani, and P.K. Wellauer. 1980. Tissue specific expression of mouse $\alpha$-amylase genes. I. Mol. Biol. 142: 93-116.
Schultz, R.M. and P.M. Wassarman. 1977a. Specific changes in the pattern of protein synthesis during meiotic maturation of mammalian oocytes in vitro. Proc. Natl. Acad. Sci. 74: $538-541$.

- 1977b. Biochemical studies of mammalian oogenesis: Protein synthesis during oocyte growth and meiotic maturation in the mouse. J. Cell Sci. 24: 167-194.

Smith, R.C., W.M. Bement, M.A. Dersch, E. Dworkin-Rastl, M. Dworkin, and D.G. Capco. 1990. Nonspecific effects of oligodeoxynucleotide injection in Xenopus oocytes: A reevaluation of previous D7 mRNA ablation experiments. Development 110: 769-779.

Strickland, S., J. Huarte, D. Belin, A. Vassalli, R.J. Rickles, and J.-D. Vassalli. 1988. Antisense RNA directed against the $3^{\prime}$ noncoding region prevents dormant mRNA activation in mouse oocytes. Science 241: 680-684.

Varnum, S.M. and W.M. Wormington. 1990. Deadenylation of maternal mRNAs during Xenopus oocyte maturation does not require specific cis-sequences: A default mechanism for translational control. Genes \& Dev. 4: 2278-2286.

Vassalli, J.-D., J. Huarte, D. Belin, P. Gubler, A. Vassalli, M.L. O'Connell, L.A. Parton, R.J. Rickles, and S. Strickland. 1989. Regulated polyadenylation controls mRNA translation during meiotic maturation of mouse oocytes. Genes \& Dev. 3: 2163-2171.

Wickens, M. 1990. In the beginning is the end: Regulation of poly(A) addition and removal during early development. Trends Biochem. Sci. 15: 320-324. 


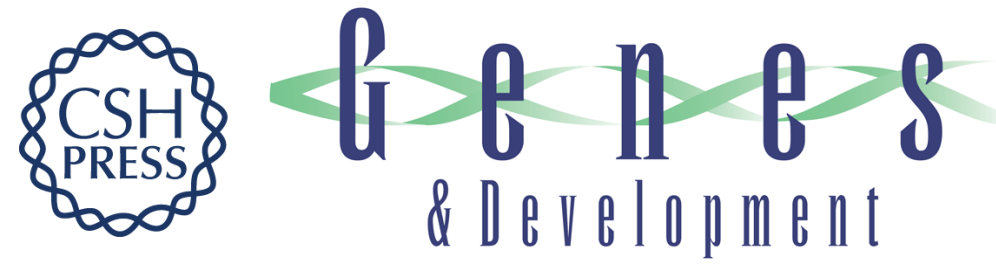

\section{Isolation of novel murine maternal mRNAs regulated by cytoplasmic polyadenylation.}

F J Sallés, A L Darrow, M L O'Connell, et al.

Genes Dev. 1992, 6:

Access the most recent version at doi:10.1101/gad.6.7.1202

References This article cites 41 articles, 21 of which can be accessed free at: http://genesdev.cshlp.org/content/6/7/1202.full.html\#ref-list-1

License

Email Alerting

Service

Receive free email alerts when new articles cite this article - sign up in the box at the top right corner of the article or click here.

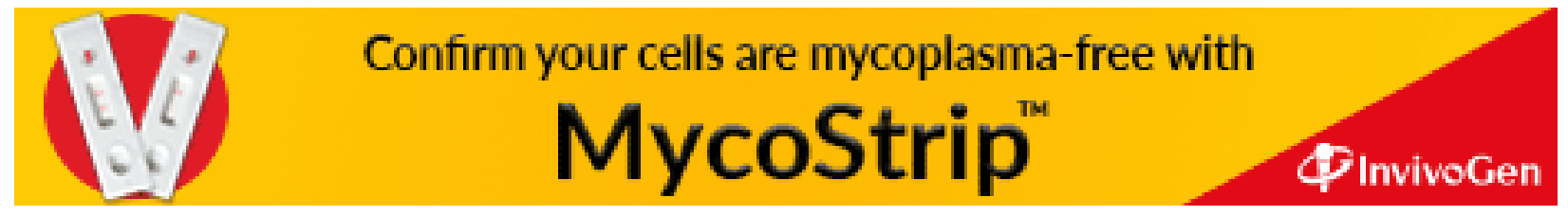

